NC-140 systems trial. J. Amer. Pomol. Soc. $5: 222-240$.

Marini, R.P., B.H. Barritt, J.A. Barden, J. Cline, R.L. Granger, M.M. Kushad, M. Parker, R.L. Perry, T. Robinson, S. Khanizadeh, and C.R. Unrath. 2001b. Performance of 'Gala' apple on eight dwarf rootstocks: Ten-year summary of the 1990 NC-140 rootstock trial. J. Amer. Pomol. Soc. 55:197-204.

Neilsen, G.H., J. Beulah, E.J. Hogue, and R.S.Utkhede. 1991. Use of greenhouse seedling bioassays to predict first year growth of apple trees planted in old orchard soil. HortScience 26:1353-1386.

Oberhofer, H. 1987. Schnitt der Schlanken Spindle. (Pruning the Slender Spindle) Sudtiroler Beratunspring fur Obst-und Weinbau. Lana, Italy. [English translation by British Columbia Ministry of Agriculture and Fisheries, Victoria, Canada, 1990.]

Perry, R.L. 1990. Training and pruning the slender spindle and vertical axis tree. Compact News 2:2-8.

Robinson, T.L., A.N. Lakso, and S.G. Carpenter. 1991. Canopy development, yield, and fruit quality of 'Empire' and 'Delicious' apple trees grown in four orchard production systems for ten years. J. Amer. Soc. Hort. Sci. 116:179-187.

Slykhuis, J.T. and T.S.C. Li. 1985. Responses of apple seedlings to biocides and phosphate fertilizers in orchard soils in British Columbia. Can. J. Plant. Pathol. 7:294-301

Slykhuis, J.T. 1990. Apple replant disease, p. 47-48. In: A.L. Jones and N.S. Aldwinkle. Compendium of apple and pear diseases. APS Press, St. Paul, Minn.

Traquair, J.A. 1984. Etiology and control of orchard replant problems: A review. Can. J. Plant Pathol. 6:54-62.

Unrath, C.R., T.B. Sutton and J.L. Whitworth. 1991. Growth and survival of apple trees replanted in established orchards as affected by preplant fumigation and postplant Ridomil, 1984-1989. Fungicide Nematic Tests 46:44.

Wertheim, S.J. 1978. Pruning of slender spindle type trees. Acta Hort. 65: 173-180

Williams, K.M. and B.H. Barritt. 1991. Intensive orchard management systems in western Europe. Good Fruit Grower 42:17-23.

Yadava, U.L. and S.L. Doud. 1980. The short life and replant problems of deciduous fruit trees. Hort. Rev. 2:1-116.

\section{Amendment of Muskmelon and Watermelon Transplant Media with Plant Growth-Promoting Rhizobacteria: Effects on Seedling Quality, Disease, and Nematode Resistance}

\author{
Nancy Kokalis-Burelle, ${ }^{1}$ \\ C.S. Vavrina, ${ }^{2}$ M.S. Reddy, ${ }^{3}$ \\ and J.W. Kloepper ${ }^{4}$
}

Additional INDEX WORDs. angular leaf spot, cantaloupes, Citrullus lanatus, Cucumis melo, gummy stem blight, root-knot nematode, transplant production

Summary. Greenhouse and field trials were performed on muskmelon (Cucumis melo) and watermelon (Citrullus lanatus) to evaluate the effects of six formulations of plant growth-promoting rhizobacteria (PGPR) that have previously been shown to increase seedling growth and induce disease resistance on other transplanted vegetables. Formulations of Gram-positive bacterial strains

This research was supported by a Cooperative Research and Development Agreement between USDA, ARS and Gustafson LLC (CRADA \#58-3K95-8-640). PGPR formulation LS2 13 has been developed into the commercial product BioYield (Gustafson LLC, Plano, Texas). Mention of trade names or commercial prodTexas). Mention of trade names or commercial prod-
ucts in this publication is solely for providing specific information and does not imply recommendation or endorsement by the U.S. Department of Agriculture. We would like to thank Karen Armbrester and Bryan Beaty for assistance in many aspects of this research.

Research ecologist, USDA, ARS, U.S. Horticultural Research Lab, 2001 S. Rock Rd., Ft. Pierce, FL, 34945. To whom reprint requests should be addressed; e-mail nburelle@saa.ars.usda.gov.

${ }^{2}$ Professor, University of Florida Institute of Food and Agricultural Sciences Southwest Florida Research \& Education Center, Immokalee, FL, 34142 .

${ }^{3}$ Associate research professor, Department of Entomology and Plant Pathology, Auburn University, AL, 36849 .

${ }^{4}$ Professor, Department of Entomology and Plant Pathology, Auburn University, AL, 36849. were added to a soilless, peat-based transplant medium before seeding. Several PGPR treatments significantly increased shoot weight, shoot length, and stem diameter of muskmelon and watermelon seedlings and transplants. Root weight of muskmelon seedlings was also increased by PGPR treatment. On watermelon, four PGPR treatments reduced angular leaf spot lesions caused by Pseudomonas syringae pv. lachrymans, and gummy stem blight, caused by Didymella bryoniae, compared to the nontreated and formulation carrier controls. One PGPR treatment reduced angular leaf spot lesions on muskmelon compared to the nontreated and carrier controls. On muskmelon in the field, one PGPR treatment reduced root-knot nematode (Meloidogyne incognita) disease severity compared to all control treatments.

$\mathrm{I}$ n the state of Florida, over 13,659 ha $(33,750$ acres $)$ of watermelons and 2,833 ha $(7,000$ acres $)$ of muskmelons were grown in 1997-98 season (Florida Department of Agriculture, 1998). Florida growers currently faced with environmental concerns, regulatory constraints, and competition from Mexico, will soon have to face the loss of methyl bromide, the soil fumigant used to control many soilborne pathogens, nematodes, insects, and weeds. The Food Quality Protection Act (FQPA) also has accelerated the removal of other chemicals used in vegetable production from the market as industry declines to reregister older pesticides. This has resulted in research efforts focused on the development of agricultural systems based on reduced chemical inputs and an increased incorporation of biological control tactics.

Plant growth-promoting rhizobacteria have been shown to enhance plant growth and protect roots from pathogens on many crops (Weller, 1988). One of these PGPR formulations, Kodiak (Gustafson LLC, Plano, Texas), is a biological seed/hopper box treatment for use in agronomic crops. Kodiak contains Bacillussubtilis (strain GB03) that has been shown to promote plant growth and increase yield in peanut (Arachis bypogaea) (Turner and Backman, 1991) and cotton (Gossypium hirsutum) (Brannen and Backman, 1993, 1994).

Soilless transplant growth mixes are an ideal medium for delivery of PGPR in transplanted crops. This ap- 
proach has enabled the establishment of beneficial rhizosphere microflora and resulted in enhanced plant growth and control of soilborne pathogens in tomato (Lycopersicon esculentum) and pepper (Capsicum annuum) (KokalisBurelle et al., 2002). Previous work with a formulation of $B$. subtilis and B. amyloliquefaciens (LS213) in muskmelon and watermelon (Vavrina, 1999) resulted in increased plant growth in the greenhouse, disease suppression in the field and improved yields. PGPR formulation LS213, which is included in these studies, has recently been developed into the commercial product BioYield (Gustafson LLC).

The objective of this research was to apply several combination treatments, including GBO3 and other Bacillus spp. known to have growth promotion and/or disease reduction capabilities, to the soilless growth medium used in the production of watermelon and muskmelon transplants. Effects of treatments on plant growth and disease prevention in greenhouse trials, and on plant growth, disease prevention and yield in field trials at three diverse locations were assessed.

\section{Materials and methods}

Transplant PRoduction. All trials included nine treatments: six bacterial treatments, two formulation carrier controls, and a nontreated control. The two carrier controls consisted of chitin (Sigma Chemical, St. Louis) at $1 \%$ and $2.5 \%$. The six bacterial treatments were LS290 (Bacillus subtilis strain $\mathrm{GBO} 3+$ B. amyloliquefaciens strain IN937a at $10^{8} \mathrm{CFU} / \mathrm{L}$ in $1 \%$ carrier), LS213 (B. subtilisstrain GBO3 + B. amyloliquefaciensstrain IN937a at $10^{9} \mathrm{CFU} / \mathrm{Lin} 2.5 \%$ carrier $), \mathrm{LS} 254$ ( $B$. subtilisstrain GBO3 + B. pumilusstrain SE34 at $10^{9} \mathrm{CFU} / \mathrm{L}$ in $2.5 \%$ carrier), LS255 (B. subtilis strain GBO3 + B. subtilis strain IN937b at $10^{9} \mathrm{CFU} / \mathrm{Lin}$ $2.5 \%$ carrier), LS256 (B. subtilis strain GBO3 + B. pumilus strain INR7 at $10^{9} \mathrm{CFU} / \mathrm{L}$ in $2.5 \%$ carrier), LS257 (B. subtilis strain GBO3 + B. pumilus strain $\mathrm{T} 4$ at $10^{\circ} \mathrm{CFU} / \mathrm{L}$ in $2.5 \%$ carrier). Bacillus subtilis strain GBO3 was supplied by Gustafson LLC while the six companion strains were supplied by the phytobacteriology lab at Auburn University (J. W. Kloepper). All bacterial and carrier control treatments were added to a soilless medium $(70 \%$ peat, $30 \%$ vermiculite, Speedling PeatLite; Speedling Inc., Sun City, Fla.) at a ratio of $1: 40(\mathrm{v} / \mathrm{v})$ and placed into 128 cell Speedling flats immediately before seeding.

'Athena' muskmelon and 'Star Gazer' watermelon cultivars (Asgrow Seed, Kalamazoo, Mich.) were used. Transplants were grown at the Southwest Florida Research and Education Center in Immokalee, (SWFREC) in accordance with south Florida standards for commercial production (Vavrina et al., 1998). Plants were fertilized weekly with $100 \mathrm{mg} \cdot \mathrm{L}^{-1}$ $(\mathrm{ppm})$ of nitrogen $(\mathrm{N})$ with soluble $20 \mathrm{~N}-8.6 \mathrm{P}-16.6 \mathrm{~K}$ with $0.1 \%$ magnesium $(\mathrm{Mg}), 0.02 \%$ boron (B), copper $(\mathrm{Cu})$, iron $(\mathrm{Fe})$, manganese $(\mathrm{Mn})$ and zinc $(\mathrm{Zn})$ and $0.0005 \%$ molybdenum (Mo), (Peters Professional Soluble Plant Food; Scotts-Sierra Horticultural Products Co., Marysville, Ohio). Treatments were replicated four times within a production greenhouse with each 128-cell flat representing one replication. Treatments were arranged in a completely randomized design on the greenhouse bench. Upon completion of the test ( 6 weeks), five seedlings per replication per treatment were sampled and assessed for dry weights of roots, dry weights of stems and leaves, stem length, stem diameter, and number of true leaves. The remaining seedlings were used for nematode screening, foliar pathogen screening, and field trials.

Nematode screening. Greenhouse experiments were conducted during 1999 and repeated in 2000 at the USDA-ARS, U.S. Horticultural Research Laboratory, Ft. Pierce, Fla., to evaluate the PGPR treatments for suppression of root-knot nematode. The root-knot nematode isolate used originated from Sanford, Fla., and was maintained on tomato in field soil before use. Infested field soil was thoroughly mixed in a cement mixer 1:1 with clean builder's sand and placed in $10.2 \times 10.2-\mathrm{cm}$ (4-inch) pots. One muskmelon or watermelon seedling was planted per pot. Treatments were replicated eight times and were arranged in randomized complete blocks. After 4 weeks, plants were assessed for root galling and general root condition. Root galling was assessed using a root gall index based on a scale of 1 to 10 with $1=$ no galls and $10=$ $100 \%$ of root system affected by galls (Zeck, 1971). Subjective root ratings were performed utilizing a 1 to 5 scale for root condition where $1=0 \%$ to $20 \%$ discolored roots, $2=21 \%$ to $40 \%, 3$ $=41 \%$ to $60 \%, 4=61 \%$ to $80 \%, 5=$ $81 \%$ to $100 \%$.

Foliar PATHOGEN SCREENING. Disease screening for induced resistance to foliar pathogens was performed in 1999 and repeated in 2000 using seedlings produced for field trials. Foliar pathogen screening experiments were performed in the Plant Sciences Research Center greenhouses at $\mathrm{Au}-$ burn University. Pathogens used for the challenge inoculation were Didymella bryoniae, causal agent of gummy stem blight (GSB) and Pseudomonassyringae pv. lachrymans, causal agent of angular leaf spot (ALS).

Pseudomonas syringae pv. lachrymanswas maintained at $-80^{\circ} \mathrm{C}(-112.0$ $\left.{ }^{\circ} \mathrm{F}\right)$ in tryptic soy broth amended with $20 \%$ glycerol before use in the challenge inoculation. Inoculum for experiments was prepared by streaking the pathogen onto tryptic soy agar plates, incubating plates at $29.0^{\circ} \mathrm{C}\left(85^{\circ} \mathrm{F}\right)$ for $24 \mathrm{~h}$, and scraping bacterial cells from plates in sterile distilled water, to yield $10^{7}$ $\mathrm{CFU} / \mathrm{mL}$. The isolate of $D$. bryoniae was maintained on potato dextrose agar (PDA) before use. Inoculum of D. bryoniae was produced on PDA plates by allowing cultures to grow for 3 weeks at room temperature. After 3 weeks, cultures on PDA plates were flooded with sterilized distilled water and gently scraped to release conidia from pycnidia. The suspension was filtered through four layers of sterile cheesecloth. The spore concentration was determined by counting in a hemocytometer and was adjusted to $10^{5}$ conidia/mL

Seedlings were transplanted into $10.2 \times 10.2-\mathrm{cm}$ plastic pots containing soilless growth medium (Speedling PeatLite, $70 \%$ peat, $30 \%$ vermiculite). Plants were inoculated 12 to $13 \mathrm{~d}$ after transplanting with a suspension of either ALS pathogen or GSB pathogen for disease evaluations. Pathogens were sprayed on the entire seedling until run-off. After inoculation, plants were placed in a humidity chamber $(100 \%$ relative humidity) for $48 \mathrm{~h}$ before placing them on a growth chamber bench. Treatments were replicated 10 times and arranged in randomized complete blocks. The plants were fertilized with Peter's solution $(20 \mathrm{~N}-8.6 \mathrm{P}-16.6 \mathrm{~K})$ once a week and watered daily. All experiments were performed at 25.0 to $26.0^{\circ} \mathrm{C}\left(77\right.$ to $\left.79{ }^{\circ} \mathrm{F}\right)$ with $14 \mathrm{~h}$ incandescent light per day. Seven days 
after ALS inoculation and $21 \mathrm{~d}$ after GSB inoculation, disease severity was assessed by counting the total number of lesions per seedling.

Field tRials. Six field trials were conducted, four trials in Spring 1999 at two locations in Florida and two trials in Spring 2000 at one location in Alabama. One muskmelon and one watermelon study were performed at the Uniroyal Chemical Company Inc., Florida Research Station, Sanford, Fla. in Spring 1999. Fertilizer [2802.1 $\mathrm{kg} \cdot \mathrm{ha}^{-1}(2500 \mathrm{lb} / \mathrm{acre}) 8 \mathrm{~N}-0.9 \mathrm{P}-$ $9.9 \mathrm{~K}]$ was broadcast over the field before bedding and 1,3-dichloropropene $(1,3-\mathrm{D})$ was broadcast applied to the entire area at a.i. $11.3 \mathrm{~kg} \cdot \mathrm{ha}^{-1}(10$ $\mathrm{lb} /$ acre). Planting beds were $20.3 \mathrm{~cm}$ ( 8 inches $)$ in height, and $0.9 \mathrm{~m}(3 \mathrm{ft})$ wide and spaced $1.8 \mathrm{~m} \mathrm{(6ft)}$ apart. A single centrally located drip irrigation line was laid $5.1 \mathrm{~cm}$ ( 2 inches $)$ below the soil surface and coextruded white on black low-density polyethylene mulch was applied. 'Athena' muskmelon and 'Star Gazer' watermelon transplants treated with PGPR formulations as previously described were planted in Mar. 1999. The crops were fertilized by fertigation to approximate Florida Extension Service recommendations of $134.5 \mathrm{~kg} \cdot \mathrm{ha}^{-1}$ (120 lb/acre) N, 87.4 $\mathrm{kg} \cdot \mathrm{ha}^{-1}$ (78 lb/acre) P, $224.2 \mathrm{~kg} \cdot \mathrm{ha}^{-1}$ $(200 \mathrm{lb} /$ acre $)$ K. Plots were scouted and sprayed with fungicides and insecticides as needed. Six harvests were taken from muskmelon plots over a 4-week period in May and June, and one harvest was taken from watermelon plots in early June.

One muskmelon and one watermelon trial were established at the SWFREC in Spring 1999. Muskmelon transplants were planted in plastic-mulched beds that had been previously cropped with tomatoes (drip irrigated), and watermelon transplants were planted in plastic-mulched beds that had been previously cropped with pepper (seep irrigated). Muskmelon field spacing was $44.1 \mathrm{~cm}$ (18 inhes) in-row with rows on $1.8-\mathrm{m}(6 \mathrm{ft})$ centers. Watermelon in-row spacing was $0.9 \mathrm{~m}(3$ $\mathrm{ft})$ with rows on 3.7-m (12 ft) centers. To approximate Florida Extension Service recommendations ( $134.5 \mathrm{~kg} \cdot \mathrm{ha}^{-1}$ $\left.\mathrm{N}, 87.4 \mathrm{~kg} \cdot \mathrm{ha}^{-1} \mathrm{P}, 224.2 \mathrm{~kg} \cdot \mathrm{ha}^{-1} \mathrm{~K}\right)$ the crops were fertilized by either fertigation (drip irrigated muskmelon) or granular fertilizer (seep irrigated watermelon). Twelve muskmelon and 12 watermelon plants were set for each treatment. Six replications were arranged in a randomized complete block design. Mancozeb and chlorothalonil fungicides were applied to prevent the advancement of fungal diseases. Bacillus thuringiensis treatments were applied throughout the growing season to reduce Lepidopteran insect pressure. Field data on plant dry weight (one arbitrarily chosen plant per replication by treatment) were taken at 30 and $45 \mathrm{~d}$ after planting (DAP). Yield data were collected from four harvests for muskmelon and one harvest for watermelon.

One watermelon and one muskmelon trial were conducted in Spring 2000 at the Auburn University Sand Mountain Agricultural Research Station, Crossville, Ala. Fertilizer $(5 \mathrm{~N}-4.4 \mathrm{P}-12.4 \mathrm{~K})$ was broadcast applied before planting at $142.3 \mathrm{~kg} \cdot \mathrm{ha}^{-1}$ ( $127 \mathrm{lb} /$ acre), and calcium nitrate was applied at $470.7 \mathrm{~kg} \cdot \mathrm{ha}^{-1}(420 \mathrm{lb} /$ acre $)$ $30 \mathrm{~d}$ after planting. No soil fumigant or plastic mulch was applied. Both muskmelons and watermelons were planted into $11.0-\mathrm{m}(36 \mathrm{ft})$ plots, and spaced

Table 1. Effects of formulations on muskmelon and watermelon transplant growth in the greenhouse 6 weeks after seeding, Immokalee, Fla.

\begin{tabular}{|c|c|c|c|c|c|c|c|}
\hline Treatment & $\begin{array}{c}\text { Leaf } \\
\text { area } \\
\left(\mathrm{cm}^{2}\right)^{\mathrm{z}}\end{array}$ & $\begin{array}{c}\text { Fresh } \\
\text { shoot wt } \\
(\mathrm{g})^{\mathrm{y}}\end{array}$ & $\begin{array}{c}\text { Dry } \\
\text { shoot wt } \\
(\mathrm{g}) \\
\end{array}$ & $\begin{array}{c}\text { Dry } \\
\text { root wt } \\
(\mathrm{g})\end{array}$ & $\begin{array}{l}\text { Shoot } \\
\text { length } \\
(\mathrm{cm})^{x}\end{array}$ & $\begin{array}{c}\text { True } \\
\text { leaf } \\
\text { (no.) }\end{array}$ & $\begin{array}{l}\text { Stem } \\
\text { diam } \\
(\mathrm{mm})^{\mathrm{w}}\end{array}$ \\
\hline \multicolumn{8}{|l|}{ Muskmelon } \\
\hline Control & 13.41 & 2.39 & 0.1049 & 0.0404 & 6.9 & 1.1 & 3.5 \\
\hline Carrier $2.5 \%$ & $20.21^{*}$ & 2.08 & $0.1310^{*}$ & $0.0515^{*}$ & 7.4 & $1.8^{*}$ & 3.3 \\
\hline Carrier $1.0 \%$ & $17.25^{*}$ & 2.31 & 0.1166 & 0.0455 & 6.8 & $1.6^{*}$ & 3.4 \\
\hline LS 290 & $15.94^{*}$ & 2.65 & 0.1138 & $0.0463^{*}$ & 7.8 & 1.3 & $4.4^{*}$ \\
\hline LS 213 & $20.18^{*}$ & $3.13^{*}$ & $0.1252^{*}$ & $0.0484^{*}$ & $8.4^{*}$ & $1.8^{*}$ & $4.1^{*}$ \\
\hline LS 254 & $20.35^{*}$ & $2.91^{*}$ & $0.1223^{*}$ & $0.0482^{*}$ & 7.5 & $1.8^{*}$ & 3.7 \\
\hline LS 255 & $18.83^{*}$ & 2.64 & 0.1178 & 0.0442 & 7.9 & $1.8^{*}$ & 3.4 \\
\hline LS 256 & $20.69^{*}$ & $2.89^{*}$ & $0.1244^{*}$ & $0.0491^{*}$ & $9.1^{*}$ & $1.8^{*}$ & 3.3 \\
\hline LS 257 & $18.94^{*}$ & $3.09^{*}$ & $0.1188^{*}$ & $0.0492^{*}$ & $11.4^{*}$ & $1.8^{*}$ & 3.7 \\
\hline $\operatorname{LSD}^{\mathrm{v}} 5 \%$ & 2.18 & 0.31 & 0.0136 & 0.0059 & 1.1 & 0.3 & 0.3 \\
\hline \multicolumn{8}{|l|}{ Watermelon } \\
\hline Control & 12.24 & 2.11 & 0.1146 & 0.0347 & 4.4 & 1.9 & 3.6 \\
\hline Carrier $2.5 \%$ & $15.58^{*}$ & $2.48^{*}$ & 0.1155 & 0.0383 & 4.7 & 2.0 & $4.1^{*}$ \\
\hline Carrier $1.0 \%$ & 13.98 & 2.32 & 0.1157 & 0.0381 & 4.8 & 2.0 & $4.3^{*}$ \\
\hline LS 290 & 12.80 & $3.19^{*}$ & 0.1094 & 0.0362 & $7.7^{\star}$ & 2.0 & 4.0 \\
\hline LS 213 & $14.83^{*}$ & $2.49^{*}$ & 0.1186 & 0.0381 & $5.6^{*}$ & 2.0 & $4.4^{*}$ \\
\hline LS 254 & $15.27^{*}$ & $2.45^{*}$ & 0.1159 & 0.0410 & 4.7 & 2.0 & $4.5^{*}$ \\
\hline LS 255 & 13.24 & $2.81^{*}$ & 0.1041 & 0.0361 & $6.5^{*}$ & 2.0 & $4.4^{*}$ \\
\hline LS 256 & $15.47^{*}$ & $2.89^{\star}$ & 0.1181 & 0.0402 & $6.9^{*}$ & 1.9 & $4.6^{*}$ \\
\hline LS 257 & 13.95 & $2.44^{*}$ & 0.1112 & 0.0347 & $5.5^{*}$ & 2.0 & $4.4^{*}$ \\
\hline LSD $5 \%$ & 1.97 & 0.28 & NS & NS & 0.76 & NS & 0.36 \\
\hline
\end{tabular}

${ }^{2} 1.00 \mathrm{~cm}^{2}=0.155$ inch $^{2}$

$\mathrm{y} 1.00 \mathrm{~g}=0.035 \mathrm{oz}$

${ }^{\mathrm{x}} 1.0 \mathrm{~cm}=0.39$ inch.

${ }^{\mathrm{w}} 1.0 \mathrm{~mm}=0.04$ inch

"LSD = least significant difference procedure.

*Significantly different from nontreated control at $P \geq 0.05$ 
at $0.9 \mathrm{~m}(3 \mathrm{ft})$, with rows placed on 2.4-m $(8 \mathrm{ft})$ centers. Treatments were replicated six times and arranged in randomized complete blocks. Plants were watered and fertigated with supplemental $\mathrm{N}-\mathrm{P}-\mathrm{K}$ through drip irrigation. Plant growth measurements were taken at transplanting ( 10 plants / treatment $)$, at $14 \mathrm{DAP}$ ( 2 plants / plot $)$, and at 28 DAP (2 plants/plot). Yield was evaluated for eight plants per plot for both muskmelon and watermelon. Nematode evaluations were performed on two plants/plot at the end of the season (80 DAP).

Statistical analysis. Data were statistically analyzed according to standard procedures including SAS general linear model (GLM) and least significant difference (LSD) procedures (SAS, Institute, Cary, N.C.). Unless otherwise stated, all differences referred to in the text were significant at the $5 \%$ level of probability.

\section{Results}

SeedLing GRowth. Treatment of seedlings with PGPR formulations improved watermelon and muskmelon growth and vigor. In 1999, several PGPR treatments (including LS213) increased shoot weight, shoot length, and stem diameter of muskmelon seedlings compared to the nontreated control treatment (Table 1 ). In addition to several PGPR treatments, both carrier control treatments increased the number of true leaves in muskmelon (Table 1). In watermelon, the only significant increase in seedling growth with PGPR treatments compared to the nontreated and formulation control treatments was an increase in shoot length with LS2 13 (Table 1). Growth data for muskmelon and watermelon seedlings used in 2000 were similar to data collected for 1999 seedlings.

Greenhouse challenges. In nematode challenge experiments, while several PGPR treatments differed from the nontreated control for fresh and dry root weight and root condition, none were consistently better than the carrier controls (Table 2). However, for both muskmelon and watermelon, LS290 consistently improved root condition compared with the nontreated control treatment (Table 2). In muskmelon there were no treatments that reduced nematode gall ratings compared to the untreated control (Table 2). Unexpectedly, in watermelon the untreated control had the lowest nematode gall ratings and one treatment, LS290, had increased nematode galling compared to the untreated control (Table $2)$. Replicate trials on muskmelon and watermelon produced similar results.

In screening trials for induction of disease resistance against foliar pathogens, all PGPR strains on watermelon reduced angular leaf spot lesions compared to the nontreated and carrier controls (Table 3). LS254, LS255, LS256, and LS257 also reduced gummy stem blight lesions on watermelon (Table 3 ). LS290, LS213, LS254, LS255, and LS256 reduced muskmelon angular leaf spot lesions compared to the controls while LS290, LS254 and the carrier control reduced gummy stem lesions (Table 3 ). Results of disease screening trials for 2000 were similar to data obtained in 1999 trials.

Field Growth Ratings. Two weeks after transplanting, all PGPR treatments increased muskmelon plant height compared to the nontreated or carrier control treatments (Table 4). In addition, LS255 and LS256 increased muskmelon shoot dry weight compared to the nontreated control (Table 4). All PGPR treatments (except

Table 2. Effects of formulations on growth of muskmelon and watermelon seedlings in the greenhouse 10 weeks after seeding, USDA-ARS, Ft. Pierce, Fla.

\begin{tabular}{|c|c|c|c|c|c|}
\hline Treatment & $\begin{array}{c}\text { Fresh } \\
\text { root wt } \\
(\mathrm{g})^{\mathrm{z}}\end{array}$ & $\begin{array}{c}\text { Root } \\
\text { condition }^{y}\end{array}$ & $\begin{array}{l}\text { Gall } \\
\text { rate }^{\mathrm{x}}\end{array}$ & $\begin{array}{c}\text { Dry } \\
\text { root wt } \\
(\mathrm{g})\end{array}$ & $\begin{array}{c}\text { Dry } \\
\text { shoot wt } \\
(\mathrm{g})\end{array}$ \\
\hline \multicolumn{6}{|l|}{ Muskmelon } \\
\hline Control & $8.03 \mathrm{c}^{\mathrm{w}}$ & $2.92 \mathrm{a}$ & $4.40 \mathrm{ab}$ & $0.50 \mathrm{~b}$ & $3.80 \mathrm{~d}$ \\
\hline Carrier 2.5\% & $8.68 \mathrm{bc}$ & $2.74 \mathrm{a}-\mathrm{c}$ & $4.13 \mathrm{ab}$ & $0.57 \mathrm{ab}$ & $4.72 \mathrm{a}-\mathrm{d}$ \\
\hline Carrier $1.0 \%$ & $8.88 \mathrm{bc}$ & $2.77 \mathrm{ab}$ & $3.79 \mathrm{~b}$ & $0.87 \mathrm{a}$ & $5.57 \mathrm{ab}$ \\
\hline LS 290 & $10.58 \mathrm{ab}$ & $2.33 \mathrm{c}$ & $4.30 \mathrm{ab}$ & $0.67 \mathrm{ab}$ & $4.79 \mathrm{a}-\mathrm{d}$ \\
\hline LS 213 & 8.14 bc & $2.66 \mathrm{a}-\mathrm{c}$ & $4.43 \mathrm{ab}$ & $0.61 \mathrm{ab}$ & $4.25 \mathrm{~b}-\mathrm{d}$ \\
\hline LS 254 & $8.96 \mathrm{bc}$ & $2.66 \mathrm{a}-\mathrm{c}$ & $4.26 \mathrm{ab}$ & $0.49 \mathrm{~b}$ & $4.01 \mathrm{~cd}$ \\
\hline LS 255 & $9.56 \mathrm{bc}$ & $2.61 \mathrm{a}-\mathrm{c}$ & $4.45 \mathrm{ab}$ & $0.84 \mathrm{a}$ & $5.41 \mathrm{a}-\mathrm{c}$ \\
\hline LS 256 & $8.77 \mathrm{bc}$ & $2.61 \mathrm{a}-\mathrm{c}$ & $4.36 \mathrm{ab}$ & $0.58 \mathrm{ab}$ & $5.74 \mathrm{a}$ \\
\hline LS 257 & $12.11 \mathrm{bc}$ & $2.42 \mathrm{bc}$ & $4.77 \mathrm{a}$ & $0.71 \mathrm{ab}$ & $3.59 \mathrm{~d}$ \\
\hline LSD $^{v} 5 \%$ & 2.45 & 0.44 & 0.72 & 0.30 & 1.49 \\
\hline \multicolumn{6}{|l|}{ Watermelon } \\
\hline Control & $2.78 \mathrm{ab}$ & $2.57 \mathrm{bc}$ & $1.30 \mathrm{~b}$ & $0.24 \mathrm{a}$ & $4.24 \mathrm{~b}$ \\
\hline Carrier $2.5 \%$ & $2.66 \mathrm{a}-\mathrm{c}$ & $2.66 \mathrm{ab}$ & $1.90 \mathrm{ab}$ & $0.21 \mathrm{c}$ & $4.83 \mathrm{ab}$ \\
\hline Carrier $1.0 \%$ & $2.49 \mathrm{bc}$ & $2.70 \mathrm{ab}$ & $2.10 \mathrm{ab}$ & $0.23 \mathrm{a}-\mathrm{c}$ & $4.91 \mathrm{a}$ \\
\hline LS 290 & $3.03 \mathrm{a}$ & $2.47 \mathrm{c}$ & $3.10 \mathrm{a}$ & $0.24 \mathrm{ab}$ & $4.35 \mathrm{ab}$ \\
\hline LS 213 & $2.56 \mathrm{bc}$ & $2.72 \mathrm{a}$ & $1.60 \mathrm{ab}$ & $0.22 \mathrm{a}-\mathrm{c}$ & $4.73 \mathrm{ab}$ \\
\hline LS 254 & $2.61 \mathrm{a}-\mathrm{c}$ & $2.64 \mathrm{ab}$ & $2.70 \mathrm{ab}$ & $0.23 \mathrm{a}-\mathrm{c}$ & $4.44 \mathrm{ab}$ \\
\hline LS 255 & $2.55 \mathrm{bc}$ & $2.57 \mathrm{bc}$ & $2.20 \mathrm{ab}$ & $0.23 \mathrm{a}-\mathrm{c}$ & $4.70 \mathrm{ab}$ \\
\hline LS 256 & $2.35 \mathrm{c}$ & $2.63 \mathrm{ab}$ & $1.90 \mathrm{ab}$ & $0.21 \mathrm{bc}$ & $4.39 \mathrm{ab}$ \\
\hline LS 257 & $2.63 \mathrm{a}-\mathrm{c}$ & $2.64 \mathrm{ab}$ & $2.50 \mathrm{ab}$ & $0.23 \mathrm{a}-\mathrm{c}$ & $4.24 \mathrm{~b}$ \\
\hline LSD 5\% & 0.42 & 0.14 & 1.78 & 0.03 & 0.61 \\
\hline
\end{tabular}

$1.00 \mathrm{~g}=0.035 \mathrm{oz}$

$\mathrm{r}$ Root condition rating: $0=$ no root rot, $1=1 \%$ to $20 \%$ discolored roots, $2=21 \%$ to $40 \%, 3=41 \%$ to $60 \%, 4=61 \%$ to $80 \%, 5=81 \%$ to $100 \%$.

'Nematode disease severity was rated on Zeck's scale of 1 to 10 , where $1=$ no galls and $10=100 \%$ galls.

"Mean of eight replications. Mean values in each column followed by the same letter are not significantly different $(P \geq 0.05)$.

${ }^{\mathrm{V}} \mathrm{LSD}=$ least significant difference procedure. 


\section{Research Reports}

Table 3. Induced systemic resistance activity of treatments against angular leaf spot and gummy stem blight of watermelon and muskmelon under greenhouse conditions, Auburn University, Ala ${ }^{z}$.

\begin{tabular}{|c|c|c|c|c|}
\hline \multirow[b]{2}{*}{ Treatment } & \multicolumn{2}{|c|}{$\begin{array}{c}\text { Watermelon } \\
\end{array}$} & \multicolumn{2}{|c|}{$\begin{array}{c}\text { Muskmelon } \\
\end{array}$} \\
\hline & $\begin{array}{l}\text { Angular leaf spot } \\
\text { (lesions/plant) }\end{array}$ & $\begin{array}{c}\text { Gummy stem blight } \\
\text { (lesions/plant) }\end{array}$ & $\begin{array}{l}\text { Angular leaf spot } \\
\text { (lesions/plant) }\end{array}$ & $\begin{array}{c}\text { Gummy stem blight } \\
\text { (lesions/plant) }\end{array}$ \\
\hline Nontreated control & $125.0 \mathrm{e}^{\mathrm{y}}$ & $74.8 \mathrm{~b}$ & $151.0 \mathrm{c}$ & $90.6 \mathrm{c}$ \\
\hline Carrier control $(2.5 \%)$ & $111.4 \mathrm{de}$ & $84.2 \mathrm{~b}$ & $125.0 \mathrm{bc}$ & $64.4 \mathrm{abc}$ \\
\hline Carrier control $(1.0 \%)$ & $115.4 \mathrm{de}$ & $73.2 \mathrm{~b}$ & $144.0 \mathrm{c}$ & $49.2 \mathrm{ab}$ \\
\hline LS290 & $81.0 \mathrm{bc}$ & $74.4 \mathrm{~b}$ & $108.6 \mathrm{ab}$ & $40.6 \mathrm{ab}$ \\
\hline LS213 & $76.8 \mathrm{bc}$ & $68.2 \mathrm{~b}$ & $81.2 \mathrm{a}$ & $55.4 \mathrm{abc}$ \\
\hline LS254 & $66.2 \mathrm{ab}$ & $33.6 \mathrm{a}$ & $83.4 \mathrm{ab}$ & $32.6 \mathrm{a}$ \\
\hline LS255 & $92.2 \mathrm{~cd}$ & $34.0 \mathrm{a}$ & $103.4 \mathrm{ab}$ & $76.4 \mathrm{bc}$ \\
\hline LS256 & $44.8 \mathrm{a}$ & $27.0 \mathrm{a}$ & $108.8 \mathrm{ab}$ & $55.2 \mathrm{abc}$ \\
\hline LS257 & $93.0 \mathrm{~cd}$ & $31.0 \mathrm{a}$ & $138.8 \mathrm{c}$ & $61.8 \mathrm{abc}$ \\
\hline $\operatorname{LSD}^{\mathrm{x}} 5 \%$ & 25.39 & 21.03 & 29.13 & 40.77 \\
\hline
\end{tabular}

${ }^{\mathrm{z}}$ Lesions per plant are means of five replications, one seedling per replication.

'Mean values in each column followed by the same letter are not significantly different $(P \geq 0.05)$.

${ }^{x} \mathrm{LSD}=$ least significant difference procedure.

Table 4. Effects of treatments on growth of muskmelon and watermelon transplants in 2000 after 2 weeks under field conditions, Crossville, $\mathrm{Ala}^{\mathrm{z}}$.

\begin{tabular}{|c|c|c|c|c|c|c|c|c|}
\hline \multirow[b]{2}{*}{ Treatments } & \multicolumn{2}{|c|}{$\mathrm{Ht}(\mathrm{cm})^{\mathrm{y}}$} & \multicolumn{2}{|c|}{ Caliper $(\mathrm{mm})^{\mathrm{x}}$} & \multicolumn{2}{|c|}{ Shoot fresh wt $(g)^{w}$} & \multicolumn{2}{|c|}{ Shoot dry wt (g) } \\
\hline & Muskmelon & Watermelon & Muskmelon & Watermelon & Muskmelon & Watermelon & Muskmelon & Watermelon \\
\hline Nontreated control & $10.7 \mathrm{a}^{\mathrm{v}}$ & $5.3 \mathrm{a}$ & $3.5 \mathrm{a}$ & $3.8 \mathrm{a}$ & $4.79 \mathrm{a}$ & $3.58 \mathrm{a}$ & $0.68 \mathrm{a}$ & $0.54 \mathrm{a}$ \\
\hline Carrier control $(2.5 \%)$ & $10.8 \mathrm{a}$ & $6.1 \mathrm{ab}$ & $4.1 \mathrm{~b}$ & $4.1 \mathrm{a}$ & $5.50 \mathrm{a}$ & $4.91 \mathrm{~b}$ & $0.82 \mathrm{abc}$ & $0.66 \mathrm{ab}$ \\
\hline Carrier control (1.0\%) & $11.9 \mathrm{ab}$ & $7.8 \mathrm{cde}$ & $4.1 \mathrm{~b}$ & $4.1 \mathrm{a}$ & $5.18 \mathrm{a}$ & $3.49 \mathrm{a}$ & $0.96 \mathrm{c}$ & $0.50 \mathrm{a}$ \\
\hline LS290 & $13.1 \mathrm{bc}$ & $7.8 \mathrm{cde}$ & $3.8 \mathrm{ab}$ & $4.5 \mathrm{~b}$ & $6.15 \mathrm{abc}$ & $5.46 \mathrm{bc}$ & $0.79 \mathrm{abc}$ & $0.80 \mathrm{bc}$ \\
\hline LS213 & $13.5 \mathrm{c}$ & 7.8 cde & $3.9 \mathrm{~b}$ & $4.6 \mathrm{~b}$ & $6.28 \mathrm{abc}$ & $6.29 c$ & $0.79 \mathrm{abc}$ & $0.94 \mathrm{~cd}$ \\
\hline LS254 & $13.1 \mathrm{bc}$ & $8.1 \mathrm{de}$ & $4.1 \mathrm{~b}$ & $4.5 \mathrm{~b}$ & $5.67 \mathrm{ab}$ & $6.05 \mathrm{bc}$ & $0.78 \mathrm{abc}$ & $0.77 \mathrm{abc}$ \\
\hline LS255 & $13.6 \mathrm{c}$ & $6.7 \mathrm{bc}$ & $4.1 \mathrm{~b}$ & $4.5 \mathrm{~b}$ & $7.07 \mathrm{bc}$ & $7.69 \mathrm{~d}$ & $0.96 c$ & $1.04 \mathrm{~d}$ \\
\hline LS256 & $13.5 \mathrm{c}$ & $8.6 \mathrm{e}$ & $3.9 \mathrm{~b}$ & $4.5 \mathrm{~b}$ & $7.46 \mathrm{c}$ & $5.13 \mathrm{bc}$ & $0.88 \mathrm{bc}$ & $0.61 \mathrm{ab}$ \\
\hline LS257 & $12.9 \mathrm{bc}$ & $6.9 \mathrm{bcd}$ & $4.0 \mathrm{~b}$ & $4.8 \mathrm{~b}$ & $5.56 \mathrm{ab}$ & $4.95 \mathrm{~b}$ & $0.70 \mathrm{ab}$ & $0.61 \mathrm{a}$ \\
\hline $\mathrm{LSD}^{\mathrm{u}} 5 \%$ & 1.2 & 1.3 & 0.3 & 0.35 & 1.51 & 1.22 & 0.19 & 0.19 \\
\hline
\end{tabular}

${ }^{2}$ Mean of six replications.

${ }^{y} 1.0 \mathrm{~cm}=0.39$ inch.

$\times 1.0 \mathrm{~mm}=0.04$ inch

${ }^{\mathrm{w}} 1.00 \mathrm{~g}=0.035 \mathrm{oz}$.

Mean values in each column followed by the same letter are not significantly different $(P \geq 0.05)$.

${ }^{\mathrm{L} S \mathrm{SD}}=$ least significant difference procedure.

LS290 on muskmelon) increased stem caliper of muskmelon and watermelon compared to the nontreated control treatment at 14 DAP(Table 4). LS290, LS213, LS254, and LS255 increased watermelon shoot dry weight compared to the nontreated and carrier controls (Table 4). In addition, all PGPR treatments (with the exception of LS255) increased watermelon top height compared to the nontreated and $2.5 \%$ carrier control treatments (Table 4). All PGPR treatments on watermelon and LS255 and LS256 on muskmelon increased shoot fresh weight compared to the nontreated and carrier control treatments (Table 4). At 28 DAP, there were no differences in muskmelon stem caliper, while all PGPR treatments continued to improved stem caliper of watermelon compared to the nontreated and carrier control treatments (data not shown).

PGPR treatments did not sig-

Table 5. Effect of treatments on suppression of nematode disease severity in muskmelon and watermelon under field conditions at the end of the season 2000 in Crossville, $\mathrm{Ala}^{\mathrm{z}}$.

\begin{tabular}{lcc}
\hline & \multicolumn{2}{c}{ Disease severity $^{\mathrm{y}}$} \\
\cline { 2 - 3 } Treatment & Muskmelon & Watermelon \\
\hline Nontreated control & $8.6 \mathrm{~cd}^{\mathrm{x}}$ & $5.9 \mathrm{c}$ \\
Carrier control (2.5\%) & $8.5 \mathrm{~cd}$ & $5.0 \mathrm{bc}$ \\
Carrier control (1.0\%) & $7.7 \mathrm{bc}$ & $4.5 \mathrm{abc}$ \\
LS290 & $7.3 \mathrm{bc}$ & $3.9 \mathrm{ab}$ \\
LS213 & $6.7 \mathrm{~b}$ & $4.2 \mathrm{abc}$ \\
LS254 & $3.9 \mathrm{a}$ & $4.0 \mathrm{abc}$ \\
LS255 & $7.9 \mathrm{bc}$ & $4.7 \mathrm{abc}$ \\
LS256 & $9.5 \mathrm{~d}$ & $2.8 \mathrm{a}$ \\
LS257 & $9.7 \mathrm{~d}$ & $4.5 \mathrm{abc}$ \\
LSD $5 \%$ & 1.3 & 1.9 \\
\hline
\end{tabular}

${ }^{2}$ Mean of six replications, two plants per replication.

'Nematode disease severity was rated on Zeck's scale of 1 to 10 , where $1=$ no galls and $10=100 \%$ galled. 'Mean values in each column followed by the same letter are not significantly different $(P \geq 0.05)$. ${ }^{w_{L S D}}=$ least significant difference procedure.

nificantly improve plant dry weight data from the Immokalee field trial. However, trends for both watermelon and muskmelon were positive. For example, all treatments had increased watermelon plant dry weight compared to the nontreated control at both 2 and 4 weeks after transplanting. 
LS256, LS257, LS290, and the 2.5\% carrier control had higher muskmelon plant dry weight than the nontreated control at both sample times (data not shown).

Field disease ratings. No significant differences in root ratings occurred among treatments on muskmelon in the Sanford, Fla., trials. Although not statistically significant, LS256 and LS290 had higher root weights, lower root condition ratings, and lower gall ratings on muskmelon compared to the $2.5 \%$ carrier controls (data not shown). No significant differences in watermelon root ratings occurred among treatments in the Sanford trial, although LS213 and LS255 did not have any galling by root-knot nematodes, while the nontreated control did have low levels of galling (data not shown).

At the end of the season in Alabama, formulation LS254 was the only treatment that significantly reduced root-knot nematode gall severity on muskmelon compared to the control treatments (Table 5), while both LS290 and LS256 reduced root-knot nematode gall severity on watermelon (Table 5). There were no differences in disease incidence on either watermelon or muskmelon in field trials performed in Immokalee, Fla.

YIELD. No significant differences occurred in watermelon or muskmelon yields in the Sanford, Fla. field trials, although LS257 resulted in numerically higher yield than the nontreated controls for both crops (data not shown). There were also no statistically significant differences among treatments on watermelon or muskmelon yield in the Immokalee, Fla. field trial, although LS254, LS255, LS256, and LS257 resulted in numerically higher yields for both crops compared to the nontreated control (data not shown). In the Alabama field trial, there were no significant yield increases in muskmelon associated with PGPR treatments compared to the controls and, in watermelon, only LS254 had significantly higher yields than the 2.5\% carrier control and several other PGPR formulations (data not shown).

\section{Discussion}

The results reported here indicate that several PGPR formulations may be useful components in production of muskmelon and watermelon. The ultimate objective in transplant produc- tion is to generate a strong, vigorous, compact plant that will establish and grow quickly in the field and produce an optimum yield. Incorporation of these biological treatments into planting media resulted in significant increases in leaf area, number of leaves, shoot weight, stem diameter, and sometimes root weight, compared to nontreated control plants. Consequently, certain PGPR formulations could be used to produce a standard sized transplant in less time or to produce a more vigorous transplant in the time currently used for standard transplant production. Increased stem diameter and root weight is indicative of strong plants that generally exhibit less susceptibility to transplant shock, drought, and attack by fungal pathogens, nematodes and insects early in the season (Vavrina, 1996).

The beneficial effects of the carrier controls on transplant growth and disease incidence may be due to the reported ability of chitin (the principle component of the carrier) to induce low levels of disease resistance (Benhamou et al., 1998). Chitin has also been reported to favor the development of nematode antagonistic microflora in soil (Rodríguez-Kábana et al., 1987), and can function as a slow release source of nitrogen. Any or all of these factors could account for the beneficial effects of the carrier control treatments in these experiments. An additional benefit of the formulation carrier functioning as a slow release nitrogen source is the potential to reduce fertilizer rates, or the number of fertilizer applications during transplant production, thereby reducing cost and pollution-causing runoff.

The benefits of PGPR treatments seen in these trials included increased root weight, improvement in root ratings, reduction of galling by root-knot nematodes, reductions in incidence of disease caused by important foliar pathogens, and modest yield increases in one watermelon trial. Nemec et al. (1996) found that a variety of biological control agents including B. subtilissuccessfully colonized roots of seedlings and some provided protection against soilborne pathogens of tomato in the field. Yield response to PGPR formulations in melons was not as substantial as those found in tomato and pepper where some PGPR formulations increased both total yield and grade of harvested fruit (Kokalis-Burelle et al.,
2002). Gagné et al. (1993) was also able to demonstrate increased yield in greenhouse produced tomatoes with the addition of PGPR to seedling production media. However, yield evaluations in melons, or other crops producing large fruit, may be confounded due to high levels of variability of small plot harvests. Accurate yield assessments for both watermelon and muskmelon require that largerscale trials be conducted according to commercial production standards.

For any particular crop, the precise value of stronger transplants will only be realized with several years of largescale field trials. However, in the four experimental field trials conducted in this study, beneficial effects of PGPR treatments where apparent and included significant decreases in several important diseases in both greenhouse and field environments. These results are supported by recent work by Egel (2000) who found a reduction in rootknot nematode damage on watermelon with LS213. Egel also demonstrated that when LS213 was combined with 1,3-D and chloropicrin combinations as a soil fumigant, total yield was increased.

Treatment of transplants with PGPR has good potential for use with many different crops, and in many different production systems, especially where pest control options are limited. The first step in maximizing productivity in transplanted crops is by improving the quality of the transplant. Providing plants with resources to achieve optimum growth and vigor will lead to better establishment of the crop.

\section{Literature cited}

Benhamou, N., J.W. Kloepper, and S. Tuzun. 1998. Induction of resistance against fusarium wilt of tomato by combination of chitosan with an endophytic bacterial strain: Ultrastructure and cytochemistry of the host response. Planta 204:153-168.

Brannen, P.M. and P.A. Backman. 1993 Cotton colonization by Bacillus subtilis inoculants to augment seedling disease control and promote season-long root health, p. 194-196 In: Proceedings of the 1993 Beltwide Cotton Production Research Conference, 13-14 Jan. 1993, New Orleans, La.

Brannen, P.M. and P.A. Backman. 1994 Suppression of fusarium wilt of cotton with Bacillus subtilis hopper box formulations, p. 83-85 In: M.H. Ryder, P.M. Stephens, and G.D. Bowen (eds.). Improving plant 
productivity with rhizosphere bacteria. Proc. 3rd Intl. Wrkshp. Plant GrowthPromoting Rhizobacteria. CSIRO Press, Adelaide, South Australia.

Egel, D.S. 2000. Evaluation of fumigants for the control of fusarium wilt and rootknot nematode of watermelon. Fungicide Nematicide Test Rpt. 2001:N19.

Florida Department of Agriculture. 1998. Florida agricultural statistics. Fla. Agr. Stat. Serv., Orlando.

Gagné, S., L. Dehbi, D. Le Quéré, F. Cayer, J-L Morin, R. Lemay, and N. Fournier. 1993. Increase of greenhouse tomato fruit yields by plant growth-promoting rhizobacteria (PGPR) inoculated into the peat-based growing media. Soil Biol. Biochem. 25:269-272.

Kokalis-Burelle, N., C.S. Vavrina, E.N Rosskopf, and R.A. Shelby. 2002. Field evaluation of plant growth-promoting rhizobacteria amended transplant mixes and soil solarization for tomato and pepper production in Florida. Plant Soil 238: 257-266.

Nemec, S., L.E. Datnoff, and J. Strandberg. 1996. Efficacy of biocontrol agents in planting mixes to colonize plant roots and control root diseases of vegetables and citrus. Crop Protection 15:735-742.

Rodríguez-Kábana, R., G. Morgan-Jones, and I. Chet. 1987. Biological control of nematodes: Soil amendments and microbial antagonists. Plant Soil 100:237-247.

Turner, J.T. and P.A. Backman. 1991. Factors relating to peanut yield increase after seed treatment with Bacillus subtilis. Plant Dis. 75:347-353

Vavrina, C.S. 1996. An introduction to the production of containerized vegetable transplants. Univ. Fla. Coop. Ext. Serv. Bul. 302.

Vavrina, C.S. 1999. The effects of LS213 (Bacilluspumilus) on plant growth promotion and systemic acquired resistance in muskmelon and watermelon transplants and subsequent field performance. Proc. Intl. Symp. Stand Establishment. p. 107-111.

Vavrina, C.S., G.J. Hochmuth, J.A. Cornell, and S.M. Olson. 1998. Nitrogen fertilization of Florida-grown tomato transplants: seasonal variation in greenhouse and field performance. HortScience 33:251-254.

Weller, D.M. 1988. Biological control of soilborne pathogens in the rhizosphere with bacteria. Annu. Rev. Phytopathol. 26:379-407.

Zeck, W.M. 1971. A rating scheme for field evaluation of root-knot nematode infestation. Pflanzenschutz 24:141-144.

\section{Arbuscular Mycorrhiza and Growth Responses of Several Ornamental Plants Grown in Soilless Peat-based Medium Amended with Coconut Dust (Coir)}

\author{
R.G. Linderman ${ }^{1}$ and
}

E.A. Davis ${ }^{2}$

ADDITIONAL INDEX WORDS. endomycor rhiza, potting mixes, marigold, minirose, zinnia, germander, lavender

SUMmary. Coconut fiber dust (coir) is being used as a peat substitute or amendment to potting mixes with varied results. However, its microbial composition and compatibility with beneficial microbes that might be added to growth media in the nursery, such as mycorrhizal fungi, has not been determined. In this study, coir was amended to a peat-based medium $(15 \%, 30 \%, 45 \%$, and $60 \%$ by volume) to determine its effects on growth of several ornamental plants and on the formation and function of the arbuscular mycorrhizal (AM) fungus Glomus intraradices. Mycorrhizae formed as well, and usually better, in all the coir-amended peat treatments as in peat alone. The magnitude of growth enhancement due to mycorrhizae was small for the plants tested in these media compared to that which usually occurs in soil-based media. In this experiment, plant growth responses appeared to be independent of level of mycorrhizal colonization and were plant species dependent. Consistent growth enhancement from mycor-

USDA, Agricultural Research Service, Horticultural Crops Research Laboratory, 3420 N.W. Orchard Avenue, Corvallis, OR 97330

The authors gratefully acknowledge the technical assistance of Karin M. Rohland. Mention of a trademark, proprietary product, or vendor does not constitute a guarantee or warranty of the product by the U. S. Department of Agriculture and does not imply its approval to the exclusion of other products or vendors that also may be suitable.

${ }^{1}$ Research plant pathologist, corresponding author; e-mail lindermr@science.oregonstate.edu.

${ }^{2}$ Faculty research assistant, Dept. of Botany and Plant Pathology. Oregon State University. rhizae only occurred with marigold (Tagetes patula). With germander (Teucrium fruticans), growth was depressed with mycorrhizal inoculation in the medium composed of $60 \%$ coir. Growth of lavender (Lavandula augustifolia) was depressed in all coir-amended media, with or without AM inoculation, compared to the nonamended control. These results confirm previous reports of varied response of plant species to coir, and indicate the lack of any detrimental effects of coir on mycorrhiza formation.

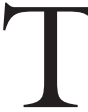
he use of coir as a suitable plant growth substrate has been reported for many greenhouse plants: rose (Rosa spp.) (Raviv et al., 2001); australian native pea (Pultenaea spp.), australian native aster (Brachycome spp.), australian native correa (Correa spp.), eucalypt (Eucalyptusspp.), spider flower (Grevillea spp.), and australian native lomandra (Lomandra spp.) (Offord et al., 1998); tickseed (Coreopsis spp.) and tomato (Lycopersicon spp.) (Pill and Ridley, 1998); arrowwood (Viburnum spp.) and lilac (Syringa spp.) (Evans and Iles, 1997); dumb cane (Dieffenbachia spp.), madagascar dragon tree (Dracaenaspp.), and peace lily (Spathiphyllum spp.) (Stamps and Evans, 1997, 1999); rhododendrons (Rhododendron spp.) (Knight et al., 1998); balsam (Impatiens spp.) (Argo and Biernbaum, 1997); jungle geranium (Ixora spp.) and star cluster (Pentas spp.) (Meerow, 1994); sunflower $(\mathrm{He}$ lianthus spp.), marigold (Tagetes spp.), petunia (Petunia $\times$ bybrida), and geranium (Pelargonium spp.) (Evans and Stamps, 1994); poinsettia (Euphorbia spp.) and lily (Lilium spp.) (Argo and Biernbaum, 1995; Waber and Evans, 1996); tailflower (Anthurium spp.) and magesty palm (Raveneaspp.)(Meerow, 1995 ).

Most reports indicate that coir is suitable for potting mixes, but reported growth responses are as varied as the plant species grown, the potting substrate mixes, the fertilizer regime, the type of peatmoss used in the mix (i.e., sphagnum versus sedge) (Meerow, 1994, 1995), and the actual sources of the coir (Abad et al., 2002; Evans et al., 1996; Konduru et al., 1999). This last factor may be one of the most crucial; the live coconut plantation site and the coir process of drying, grinding, and compression can significantly affect 